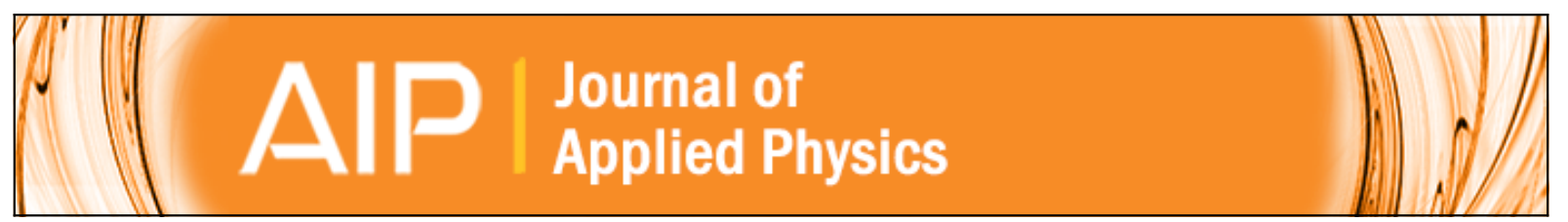

Percolation transition in the gas-induced conductance of nanograin metal oxide films with defects

Julia Dräger, Stefanie Russ, Tilman Sauerwald, Claus-Dieter Kohl, and Armin Bunde

Citation: Journal of Applied Physics 113, 223701 (2013); doi: 10.1063/1.4809572

View online: http://dx.doi.org/10.1063/1.4809572

View Table of Contents: http://scitation.aip.org/content/aip/journal/jap/113/22?ver=pdfcov

Published by the AIP Publishing

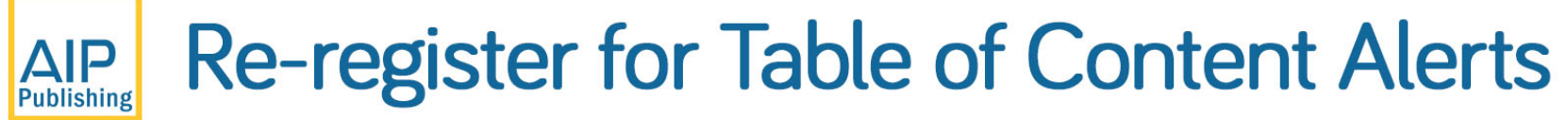




\title{
Percolation transition in the gas-induced conductance of nanograin metal oxide films with defects
}

\author{
Julia Dräger, ${ }^{1,2}$ Stefanie Russ, ${ }^{2}$ Tilman Sauerwald, ${ }^{3,4}$ Claus-Dieter Kohl, ${ }^{3}$ \\ and Armin Bunde ${ }^{1}$ \\ ${ }_{1}^{1}$ Institut für Theoretische Physik III, Justus-Liebig-Universität Giessen, D-35392 Giessen, Germany \\ ${ }^{2}$ Institut für Theoretische Physik, Freie Universität Berlin, 14195 Berlin, Germany \\ ${ }^{3}$ Institut für Angewandte Physik, Justus-Liebig-Universität Giessen, D-35392 Giessen, Germany \\ ${ }^{4}$ Lehrstuhl für Messtechnik, Universität des Saarlandes, D-66123 Saarbrücken, Germany
}

(Received 13 March 2013; accepted 21 May 2013; published online 11 June 2013)

\begin{abstract}
We use Monte-Carlo Simulations to study the conductance switching generated by gas-induced electron trapping/-releasing in films of sintered metal oxide nanoparticles by using a site-bond percolation model. We explore the possibilities of gas sensors based on these mechanisms. In our study, we model films of different thicknesses where the conductance values of the grains (sites) and of the contacts (bonds) between these grains depend on the surface density $N_{r}$ of adsorbed gas molecules from the ambient atmosphere. Below a critical density $N_{r}=N_{\mathrm{r}, \mathrm{c}}$, the system is insulating due to the interruption of current flow, either through the connecting bonds or through the grain interior. This leads to two competing critical gas covering thresholds $N_{\mathrm{r}, \mathrm{c}}^{(\mathrm{bond})}$ and $N_{\mathrm{r}, \mathrm{c}}^{(\text {(site) }}$, respectively, that separate the insulating from the conducting phase. For $N_{\mathrm{r}, \mathrm{c}}^{(\text {site })}>N_{\mathrm{r}, \mathrm{c}}^{(\mathrm{bond})}$, the characteristic curve of monodisperse sensors shows a noticeable jump from zero to a finite conductance at $N_{r}=N_{\mathrm{r}, \mathrm{c}}^{(\text {site })}$, while for polydisperse sensors site percolation effects modify the jump into a steep increase of the characteristic curve and thus lead to an enhanced sensitivity. For $N_{\mathrm{r}, \mathrm{c}}^{(\text {site })}<N_{\mathrm{r}, \mathrm{c}}^{(\text {bond) }}$, both mono- and polydisperse systems follow the same curves that show a smoother characteristic increase $\propto\left(N_{r}-N_{\mathrm{r}, \mathrm{c}}^{(\text {bond })}\right)^{2}$ which reveals that, despite the occurrence of an inherent bond percolation effect close to $N_{\mathrm{r}, \mathrm{c}}$, the increase of the bonds is the dominating effect. (C) 2013 AIP Publishing LLC. [http://dx.doi.org/10.1063/1.4809572]
\end{abstract}

\section{INTRODUCTION}

Gas sensors play an important role in our daily life, e.g., to prevent gas explosions or to control the emission of combustion gases. ${ }^{1,2}$ In this paper, we concentrate on homogeneous semiconducting gas sensors. They usually consist of a polycrystalline oxide layer that changes its electrical conductance when exposed to the gas that is therefore detected via the conductance change of the sensor. The preparation of suitable oxide layers has been considerably improved in recent years to fabricate nanogranular films by self-organized growth of nanostructures $^{3}$ as well as by the replication of nanostructure templates. ${ }^{4}$ Besides disordered nanoporous systems, also systems with long-range order and a high degree of crystallinity ${ }^{5}$ can now be used for the design of gas sensors. In addition to polydisperse systems with broad distributions of grains sizes also highly monodisperse systems can be achieved by new preparation methods. As one example, highly monodisperse granular oxide layers can now be prepared by gas phase condensation with electrostatic precipitation. ${ }^{6}$ Therefore, a large variety of complex ordered and disordered materials with monodisperse and polydisperse grain distributions can be fabricated and in principle be used as a functional film, in particular as a sensor.

Homogeneous semiconducting gas sensors usually are considered as belonging to two different classes, depending on their operating temperature. At higher temperatures, e.g., more than $500{ }^{\circ} \mathrm{C}$ for $\mathrm{TiO}_{2}, \mathrm{ZnO}, \mathrm{SnO}_{2}$, and $\mathrm{WO}_{3}$, the conductance is basically caused by oxygen vacancies inside the bulk material that act as n-donors. ${ }^{2,7}$ At lower temperatures, typically below $500{ }^{\circ} \mathrm{C}$, surface effects are considered as more important than reactions in the bulk, ${ }^{7}$ giving rise to a "surface sensitive" sensor for detecting reducing gases, as, e.g., $\mathrm{CO}, \mathrm{H}_{2}$, and hydrocarbons that adsorb on the grain surfaces, thereby also changing the conductance between the grains (see, e.g., Ref. 8). These surface-sensitive sensor types are mostly operated between $150^{\circ} \mathrm{C}$ and $350^{\circ} \mathrm{C}$ and in this paper we will concentrate on this class, namely, on the widely used surface sensitive n-type oxides as, e.g., $\mathrm{SnO}_{2}$.

It is well-known that the grain-sizes in the system play a major role for the sensing properties. ${ }^{2,4,9-12}$ When the grains are exposed to air, according to a standard model ${ }^{2,4}$ oxygen is adsorbed, captures electrons from the grain bulks and traps them at the surface (surface doping) leaving a depletion zone of thickness $\lambda$ of the order of $10 \mathrm{~nm}$ below the surface. When a reducing gas is offered, the negative surface charge is reduced and within the Schottky approximation assuming acceptor/donor compensation, $\lambda$ is given by ${ }^{13,14}$

$$
\lambda \approx\left(N_{\mathrm{ox}}-N_{r}\right) / N_{D}
$$

where $N_{r}$ and $N_{\text {ox }}$ are the surface densities of the reducing gas and the initially adsorbed oxygen, respectively. $N_{D}$ is the donor density of the metal oxide bulk material which is assumed to be constant. ${ }^{9,15}$ Equation (1) shows that $\lambda$ is the largest when the grain surface is covered solely by adsorbed oxygen, i.e., $N_{r}=0$. If $\lambda$ is of the order of the radius of the 
grain, the remaining core becomes too small to host an electron state and the conductance of the grain vanishes. When $N_{r}$ increases, $\lambda$ decreases and more and more grains eventually become conducting.

The gas sensors considered in this work consist of grains that are arranged in a disordered sintered layer and form a network between two electrodes. In this network, not only the conductance of each grain but also the conductance between overlapping grains (bonds) matters and there is only a sensing signal if there exists a conducting path between the electrodes. Based on simplifying assumptions about the network and its conducting properties, it has been shown by numerical simulations ${ }^{13,14}$ that the conductance of the gas sensor shows a percolation characteristic curve when plotting it versus $N_{r}$. Below a certain critical gas density $N_{\text {crit }}$, the system is insulating while above $N_{\text {crit }}$ the structure becomes conducting (for an overview on percolation phenomena in gas sensors see, also, Ref. 24).

On the experimental side, the influence of percolation effects on the electron transport in nanocrystalline semiconducting layers has been investigated (see Ref. 4 and references therein), e.g., by transient photocurrent measurements in nanocrystalline $\mathrm{TiO}_{2}$ solar cells ${ }^{16}$ and nanoporous $\mathrm{TiO}_{2}$ layers. ${ }^{17}$ Indications of a possible percolation mechanism in nanoporous gas sensors have also been found by gassensitivity measurements on randomly oriented single crystal $\mathrm{SnO}_{2}$ nanowires and on thin layers of pristine $\mathrm{SnO}_{2}$ nanoparticles. ${ }^{8}$ Other works have investigated the dependence of the conductance in nanocrystalline semiconducting films on various morphological system parameters, as, e.g., on thickness ${ }^{18}$ and porosity. ${ }^{17} \mathrm{~A}$ general dependence of the conductance on the system morphology has been discussed in Ref. 19.

It is the purpose of this paper to explore the possibilities of disordered homogeneous gas sensors and the specific shapes of the characteristic curves analytically and numerically. To this end, we investigate the different properties that are connected with the disordered structure and in particular with a possible percolation transition. We extend the work of Ref. 14 considerably by gradually going from a $2 \mathrm{D}$ system to a $3 \mathrm{D}$ system, by studying in detail the microscopical features that determine the shape of the characteristics and by explicitly calculating the bonds between neighboring grains. Thereby, we arrive at a site-bond percolation model and distinguish the cases with bonds between two grains being cut even when the neighboring grains are still conducting and vice versa. As a result and depending on these two cases, we find that smooth characteristic curves are possible as well as characteristic curves that jump to a finite signal at some threshold value.

The paper is organized as follows: In Sec. II, we describe the underlying model that we use in our simulations and compare it to the model used in Ref. 14. After this, we explain in Sec. III how the conductance of the whole system is estimated for a given concentration $N_{r}$ of reducing gas. In Sec. IV, we discuss a simplified monodisperse system that can be solved analytically. The results can be used as a guideline for Sec. V, where the results of our numerical simulations on mono- and polydisperse systems are presented. In Sec. VI, we discuss our results and their expected relevance to experimental situations and technical applications.

\section{MODEL}

The nanograin metal-oxide films we are interested in consist of sintered grains with connections in the shape of necks between them (see Fig. 1). It is experimentally known that the mean geometrical neck diameter $\left\langle D_{n}^{0}\right\rangle$ is proportional to the mean diameter $\langle D\rangle$ of the grains $\left\langle D_{n}^{0}\right\rangle \simeq \Theta\langle D\rangle$ with a proportionality constant $\Theta \approx 0.76$ (sintering parameter). ${ }^{9,10}$ The coordination number $\langle k\rangle$ of the grains is between 2 and 3 . In order to model such a system, we start with a cubic lattice with lattice constant $a$ consisting of $N$ monolayers and occupy the lattice points $i$ with spheres of diameter $D^{(i)}$ (with probability $p^{(\text {site) }}$ ), or leave them empty (with probability $1-p^{(\text {site })}$ ) (see Figs. 1(a) and 1(b)). The grain diameters $D^{(i)}$ may be all equal, $D^{(i)}=D$ (monodisperse), or chosen randomly from an appropriate distribution $P(D)$ (polydisperse). The lattice constant $a$ is chosen such that neighboring spheres can overlap fulfilling the constraint $\left\langle D_{n}^{0}\right\rangle \simeq \Theta\langle D\rangle$. For achieving the desired coordination number $\langle k\rangle$, we use the occupation probability $p^{(\text {site })}$ as the tuning parameter. To generate additional disorder, the center of each grain may be shifted by a random value up to $10 \%$ of the lattice constant $a$ away from the lattice point. As in realistic experimental systems, the lattice constant $a$ decreases with $\langle D\rangle$, thereby reducing the total size of the system. After generating the model grain system, it is straightforward to determine the individual grain surfaces $S_{\exp }^{(i)}$ exposed to the ambient atmosphere which is a central quantity in calculating the conductance of the system.

First, to identify the conducting grains for a given surface gas density $N_{r}$, we follow Ref. 14: we assume that the probability $p_{\mathrm{e}}^{(i)}$ of grain $i$ to be conducting is identical to the probability that it is occupied by at least one free electron. The number $\Delta N_{\text {free }}^{(i)}\left(N_{r}\right)$ is the difference between the number of the donor electrons in grain $i$ and the number of electrons trapped by adsorbed oxygen molecules

$$
\Delta N_{\text {free }}^{(i)}\left(N_{r}\right) \simeq V_{i} N_{D}-\left(N_{\mathrm{ox}}-N_{r}\right) S_{\exp }^{(i)},
$$

where $V_{i}$ is the volume of grain $i$. From Eq. (1), we obtain $p_{\mathrm{e}}^{(i)}\left(N_{r}\right)$ within a mean-field type approximation

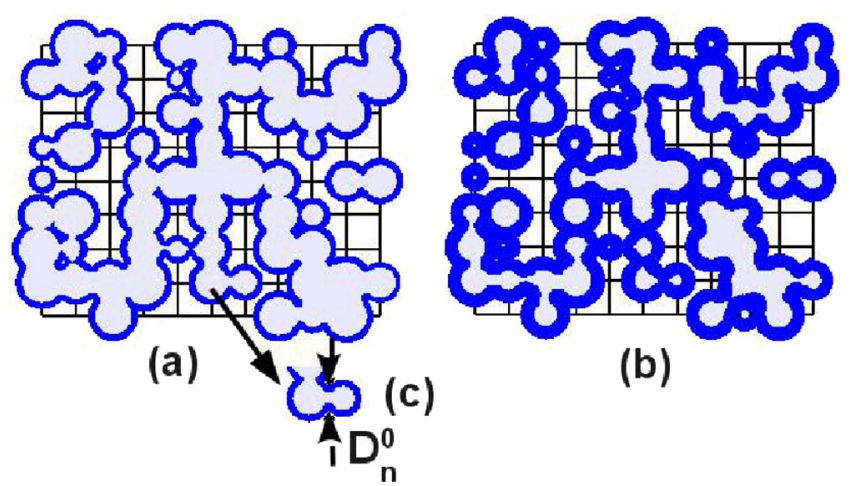

FIG. 1. (a) and (b) Two-dimensional sketch of a network model of a system of sintered nanograins with (a) small and (b) large thickness of the depletion zone $\lambda$. The conducting core region and the depletion zone are symbolized by the light blue and the dark blue shading, respectively. (c) Magnification of a single grain contact with the geometrical neck diameter $D_{n}^{0}=D_{n}\left(N_{\text {ox }}\right)$. The (smaller) diameter $D_{n}\left(N_{r}\right)$ of the conducting neck is the diameter of the light blue channel. 


$$
p_{\mathrm{e}}^{(i)}\left(N_{r}\right)= \begin{cases}0, & \Delta N_{\text {free }}^{(i)}\left(N_{r}\right) \leq 0 \\ \Delta N_{\text {free }}^{(i)}\left(N_{r}\right), & 0<\Delta N_{\text {free }}^{(i)}\left(N_{r}\right)<1 \\ 1, & \Delta N_{\text {free }}^{(i)}\left(N_{r}\right) \geq 1 .\end{cases}
$$

Second, by calculating the diameter of the conducting channel $D_{n}\left(N_{r}\right)$ between nearest-neighbor grains, we investigate for each pair of connected conducting nearest-neighbor grains $i$ and $j$ whether at the considered gas value $N_{r}$ the conducting cores of the two grains are connected. The geometrical quantity $D_{n}^{0} \equiv D_{n}\left(N_{\text {ox }}\right)$ measures the neck between two grains when $\lambda=0$. If the grains are not connected, we consider the bond between them as cleaved. Otherwise, we determine the conductance $L_{i j}$ between the grains by the "neck resistance mechanism," where the $L_{i j}$ are calculated from the area $A\left(N_{r}\right)=\pi\left(D_{n}^{0}-2 \lambda\left(N_{r}\right)\right)^{2} / 4$ of the conducting channel, the distance $d_{i j}$ between the grains and the density $N_{D}$ of charge carriers, leading to $L_{i j} \propto A\left(N_{r}\right) N_{D} / d_{i j}$. The neck resistance mechanism seems to be more relevant for smaller and well-sintered grains than possible conductance values across grain-boundaries between isolated grains. ${ }^{9}$ In the case of rough grain surfaces, the electron hopping across grain-boundaries would probably be even more suppressed, due to the confinement of the electron states. ${ }^{20}$

\section{DETERMINATION OF THE CONDUCTANCE}

Next we investigate, if a conducting path between opposite sides of the sample exists. If yes, the system is in its conducting phase and we can proceed to calculate the conductance of the system. To this end, we map the conduction problem onto the corresponding diffusion problem by defining jump probabilities $p_{i j} \propto L_{i j}$ along the bonds between nearest neighbors and calculate the mean square displacement $\left\langle r^{2}(t)\right\rangle$ of many random walkers on the infinite percolation cluster of the sample (a standard problem analyzed extensively in the literature, see, e.g., Refs. 21 and 22). According to the NernstEinstein relation, the conductance is proportional to the electron mobility $\mu$ and the density $n$ of charge carriers, where the latter scales with the number of conducting sites. We can determine $\mu$ by computing the diffusion constant: $\mu \propto D_{s}=\lim _{t \rightarrow \infty}\left\langle r^{2}(t)\right\rangle /(2 d t)$. As we calculate $\left\langle r^{2}(t)\right\rangle$ in the $x y$-plane, the dimension $d$ is equal to 2 . The time $t$ is counted by the number of time steps. In all figures of this work, we present the diffusion constant $D_{s}$ that is equivalent to the electron mobility $\mu$ (in arbitrary units). The conductance can then obtained by multiplying $\mu$ with the electron density $n$.

For each model system, we want to know how the conductance depends on the surface density of adsorbed gas atoms or molecules. We therefore decrease $N_{r}$ stepwise and compute the diffusion constant for each value of $N_{r}$, by assuming that the gas density in the whole area around the grain is in equilibrium, i.e., each grain is homogeneously surrounded by the gas. In a real experiment, this would mean that after the gas release one has to wait for each value of $N_{r}$ until equilibrium is reached. The conductance reaches a maximum when the depletion zone vanishes, i.e., when the surrounding oxygen is completely replaced by the reducing gas. When decreasing $N_{r}$, more and more oxygen molecules are adsorbed at the surface of the grains and the conducting cores of the grains become smaller, according to Eq. (1). As a result, some of the grains which were conducting before become insulating and the conductance values of the bonds decrease steadily, where some of them are even cleaved (see Fig. 1(b)). Accordingly, when passing from high to small values of $N_{r}$, we expect a dramatic drop of the conductance. In the following, we want to describe the different scenarios that finally lead to a detection limit.

\section{THEORETICAL ANALYSIS FOR MONODISPERSE SYSTEMS}

\section{A. The ordered non-diluted lattice}

To estimate the conductance via the mobility $\mu$, we first consider the simplest case where all grains have the same diameter $D$ and are located at the sites of a cubic lattice. By definition, also all individual exposed surfaces $S_{\exp }^{(i)}$ are identical, $S_{\exp }^{(i)} \equiv S_{\text {exp }}$.

First, we consider grains with large diameter $D$ that are still conducting (see Fig. 2(a)). The diameter $D_{n}$ of the conducting neck between two grains depends on the concentration of reducing gas $N_{r}$ via $\lambda$ as

$$
D_{n}\left(N_{r}\right)=D_{n}^{0}-2 \lambda\left(N_{r}\right) .
$$

The grain conductances $L\left(N_{r}\right)$ are proportional to the crosssection of the conducting overlap of the grains

$$
L\left(N_{r}\right) \sim D_{n}{ }^{2}\left(N_{r}\right)=\left(D_{n}^{0}-2 \lambda\left(N_{r}\right)\right)^{2} .
$$

By definition, the conductance as well as the electron mobility are proportional to $L\left(N_{r}\right)$ and thus

$$
\frac{\mu\left(N_{r}\right)}{\mu\left(N_{\mathrm{ox}}\right)}=\frac{L\left(N_{r}\right)}{L\left(N_{\mathrm{ox}}\right)} \text {. }
$$

According to Eq. (4), the conducting necks (bonds) between the grains become insulating (cleaved) when $2 \lambda\left(N_{\text {crit }}\right)$ $=2\left(N_{o x}-N_{\text {crit }}\right) / N_{D}=D_{n}^{0}=\Theta D$. This yields the critical

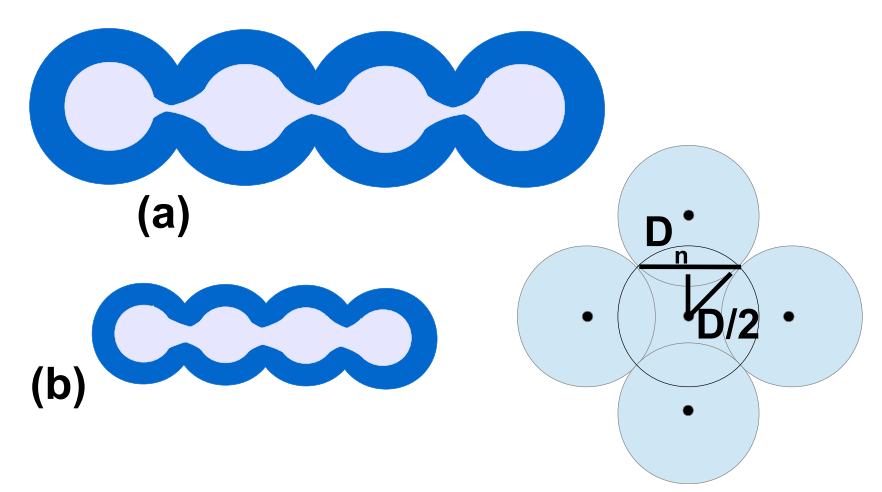

FIG. 2. (a) and (b) Sketch of the two possibilities that may lead to a breakdown of the conductance when $\lambda$ increases: (a) The bonds between large grains may become interrupted $\left(D_{n} \rightarrow 0\right)$ while the cores are still large and therefore conducting. This leads to $N_{\text {crit }}^{(\text {site }}<N_{\text {crit }}^{(\text {bond })}$. (b) The cores of small grains may become too small to host free electrons $\left(p_{\mathrm{e}}^{(i)} \ll 1\right)$ while the bonds are still conducting, leading to $N_{\text {crit }}^{\text {(bond }}<N_{\text {crit }}^{\text {(site) }}$. (c) Two-dimensional sketch for the construction of the relative free surface $s$ for the special case that next-nearest neighbors just touch each other. One easily recognizes that $\Theta=D_{n} / D=1 / \sqrt{2}$. 
gas covering threshold $N_{\text {crit }}^{\text {(bond) }}=N_{\text {crit }}$ when the conductance vanishes due to the simultaneous break-down of all bonds

$$
N_{\text {crit }}^{\text {(bond) }}=N_{\text {ox }}-\frac{\Theta N_{D}}{2} D
$$

Together with Eqs. (1), (5), and (6), we obtain the shape of the characteristic curve

$$
\mu\left(N_{r}\right)= \begin{cases}\frac{4 \mu\left(N_{\mathrm{ox}}\right)}{N_{D}^{2} \Theta^{2} D^{2}}\left(N_{r}-N_{\text {crit }}^{(\text {bond })}\right)^{2}, & N_{r}>N_{\text {crit }}^{(\text {bond })} \\ 0 & N_{r}<N_{\text {crit }}^{(\text {bond })} .\end{cases}
$$

This means that the normalized conductance $D^{2} \mu\left(N_{r}\right) / \mu\left(N_{\mathrm{ox}}\right)$ undergoes a phase transition with a universal exponent of 2 that is independent even of the lattice dimension. Also, the critical value $N_{\text {crit }}^{\text {(bond) }}$ is universal and depends-apart from the material constants $N_{D}, N_{\text {ox }}$ and $\Theta$ - only on the grain size. (Note that also $\mu\left(N_{\text {ox }}\right)$ depends on $D$.)

Second, if the grain diameter $D$ is small (and the donor density $N_{D}$ not too large), it is possible that at a critical value $N_{\text {crit }}^{(\text {site }}$ of $N_{r}$ the conducting cores of the grains become too small for hosting free electron states (see Fig. 2(b)). In this case, the conductance of the system is mainly determined by the disappearance of conducting grains which leads to an additional cut-off value $N_{\text {crit }}^{(\text {site }}$ of the characteristic curve that can be estimated in the following way: If according to Eq. (3) the value of $\Delta N_{\text {free }}\left(N_{r}\right)$ is smaller than 1, only the fraction $w_{p}\left(N_{r}\right)=\Delta N_{\text {free }}\left(N_{r}\right)$ of the grains is conducting. At a critical fraction $p_{c}^{(\text {site })}(N)$ which depends on the number $N$ of monolayers (see Sec. IV B), the conducting pathway is disrupted. Accordingly, $N_{\text {crit }}^{(\text {site }}$ can be determined from Eq. (2) by setting $\Delta N_{\text {free }}\left(N_{r}\right)=p_{c}^{(\text {site })}(N)$

$$
N_{\text {crit }}^{(\text {site })}=N_{\mathrm{ox}}-\frac{N_{D}}{6 \mathrm{~s}} D+\frac{p_{c}^{(\text {site })}(N)}{\pi \mathrm{s}} D^{-2},
$$

with the fixed fraction $\mathrm{s}=S_{\exp } /\left(\pi D^{2}\right)$ of the free surface.

In Figs. 3(a) and 3(b), we illustrate the role of the two critical gas covering thresholds $N_{\text {crit }}^{\text {(site) }}$ (Eq. (9)) and $N_{\text {crit }}^{\text {(bond) }}$ (Eq. (7)) in schematic diagrams. While $N_{\text {crit }}^{\text {(bond) }}$ defines the shape of the characteristic curve via Eq. (8), $N_{\text {crit }}^{\text {(site }}$ defines an independent cut-off value, i.e., $\mu=0$ for $N_{r}<N_{\text {crit }}^{\text {(site) }}{ }^{23}$ The conditions for the case $N_{\text {crit }}^{\text {(site) }}>N_{\text {crit }}^{\text {(bond) }}$ can be derived by subtracting Eqs. (7) and (8) yielding

$$
N_{\text {crit }}^{(\text {site })}>N_{\text {crit }}^{\text {(bond) }} \text { for }\left\{\begin{array}{cc}
D<D_{\text {crit }}^{3}, & 3 \theta s<1 \\
D>0, & 3 \theta s>1
\end{array}\right.
$$

with

$$
D_{\text {crit }}^{3}=\frac{6 p_{c}^{(\text {site })}(N)}{\pi N_{D}(1-3 \Theta \mathrm{s})}
$$

For $N_{\text {crit }}^{\text {(site) }}>N_{\text {crit }}^{\text {(bond) }}, \mu\left(N_{r}\right)$ only follows Eq. (8) for $N_{r}>$ $N_{\text {crit }}^{\text {(site) }}$ and is zero for smaller values of $N_{r}$. In this case, $N_{\text {crit }}^{\text {(site) }}$ marks a threshold value where the mobility jumps to zero (see Fig. 3(a)). For $N_{\text {crit }}^{\text {(site) }}<N_{\text {crit }}^{\text {(bond) }}$, on the other hand, the value of $N_{\text {crit }}^{\text {(site) }}$ plays no role and the characteristic curve

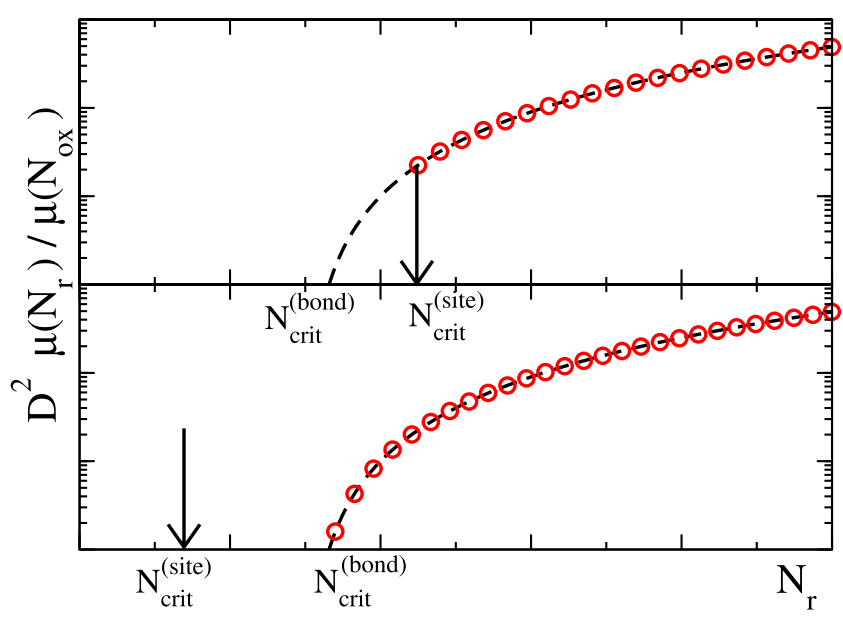

FIG. 3. (a) Sketch of the theoretical prediction for the normalized mobility $D^{2} \mu\left(N_{r}\right) / \mu\left(N_{\text {ox }}\right)$ versus $N_{r}$ for (a) $N_{\text {crit }}^{\text {(site) }}>N_{\text {crit }}^{\text {(bond }}$ and (b) $N_{\text {crit }}^{\text {(site) }}<N_{\text {crit }}^{\text {(bond) }}$. The theoretical curve for $N_{\text {crit }}^{\text {(bond) }}$ (Eq. (7)) is indicated by the dashed curve and the cut-off value $N_{\text {crit }}^{\text {(site) }}$ (Eq. (9)) by the arrow. The expected experimental values in both cases are shown by the red circles. Note the jump in the experimental values in case (a).

$\mu\left(N_{r}\right)$ follows Eq. (8) for all values of $N_{r}$ leading to a smooth curve.

One can best understand the meaning of Eq. (11) on a special example. Therefore, we calculate $D_{\text {crit }}$ exactly for a slightly smaller sinter parameter $\Theta=1 / \sqrt{2}$ where nextnearest neighbor grains just touch each other (see Fig. 2(c)), so that we can use the standard formula for the surface $A_{\text {cal }}=\pi D\left(D / 2-\sqrt{D^{2}-D_{n}^{2}} / 2\right)$ of a spherical calotte. We obtain $s=1-4 A_{\text {cal }} / \pi D^{2}=\sqrt{2}-1$ in $d=2$ and $s=1-6 A_{\text {cal }} / \pi D^{2}=3 \cdot 2^{-1 / 2}-2$ in $d=3$ so that $3 \Theta \mathrm{s}<1$ in both cases and $N_{\text {crit }}^{\text {(site) }}>N_{\text {crit }}^{\text {(bond) }}$ and $N_{\text {crit }}^{\text {(site) }}<N_{\text {crit }}^{\text {(bond) }}$ can both be achieved by choosing $N_{D}$ appropriately. The situation changes for diluted lattices, where $s$ becomes much larger so that also the case $3 \Theta s>1$ is possible.

\section{B. The diluted lattice}

In the diluted lattice, we occupy the sites with probability $p^{(\text {site })}$ by grains of fixed sizes $D$ or leave them empty with probability $1-p^{(\text {site })}$. In addition to $p^{(\text {site })}$, we again need the probability $w_{p}\left(N_{r}\right)$ that a given grain is conducting (at the gas concentration $N_{r}$ ). Due to a different number of neighbors, the individual exposed surfaces $S_{\exp }^{(i)}$ may now differ from each other. As a consequence, according to Eqs. (2) and (3), the probability of individual grains to be conducting varies. It is, however, remarkable that according to Eqs. (7) and (8), $D^{2} \mu\left(N_{r}\right) / \mu\left(N_{\text {ox }}\right)$ is unaffected by this effect and still has the same universal shape. Only the cut-off value $N_{\text {crit }}^{\text {(site) }}$ differs from the one of the non-diluted lattice as the fraction of lattice sites that host conducting grains now equals $w_{p}\left(N_{r}\right) \cdot p^{(\mathrm{site})}$. Accordingly, $N_{\text {crit }}^{(\mathrm{sit})}$ can now be determined by the condition $w_{p}\left(N_{\text {crit }}^{(\text {site) }}\right) \cdot p^{(\text {site })}=p_{c}^{(\text {site })}(N)$ which leads to a similar equation as Eq. (9), where $p_{c}^{(\text {site })}(N)$ now has to be replaced by $p_{c}^{(\text {site })}(N) / p^{\text {(site) }}$. The fraction $s$ of the free surface depends on $p^{\text {(site) }}$ and the fixed value $S_{\text {exp }}$ has to be replaced by an effective exposed surface $S_{\text {eff }}$ close to the mean value $\left\langle S_{\exp }\right\rangle$. 
While $p_{c}^{(\text {site })}(N)$ is well-known for one-layer-systems $\left(p_{c}^{(\text {site })} \simeq 0.5927\right.$ in $\left.d=2\right)$, the corresponding threshold $p_{c}^{(\text {site) }}(N)$ for an $N$ layer system $(N>1)$ decreases with the number of layers $N$ until the system eventually becomes a $3 \mathrm{~d}$-system. In order to estimate $p_{c}^{(\text {site })}(N)$ for higher $N$-values, we created percolation lattices of varying thickness $N$ by Monte-Carlo simulations and counted the number of lattices that contained an infinite percolation cluster. Figure 4 shows the fraction of this number (as compared to the number of investigated systems) as a function of $N$. The figure shows that the critical threshold $p_{c}^{(\text {site) }}(N)$ increases rapidly with $N$ towards the $3 \mathrm{~d}$ site percolation threshold $p_{c}^{\text {(site) }} \simeq 0.3116$.

With the values of $S_{\text {eff }} \approx\left\langle S_{\exp }\right\rangle$ and $p_{c}^{\text {(site) }}(N)$ from Fig. 4, we can now apply Eqs. (8) and (9) to the diluted lattice. For illustration, we plot in Fig. 5 the critical gas concentration $N_{\text {crit }}$ versus the mean grain diameter $\langle D\rangle$ for different values of $N$ (number of layers) and $p^{\text {(site) }}$ which gives us the phase diagram between the insulating and the conducting phases, where the conducting phase lies on the right-hand side of the respective critical line. The critical lines are combinations of the red solid line (that shows $N_{\text {crit }}^{\text {(bond) }}$ of Eq. (7) and is independent of $p^{(\text {site })}$ ) and the appropriate black dashed line (that shows $N_{\text {crit }}^{(\text {site }}$ of Eq. (9) and depends on $\left.p^{(\text {site })}\right)$. The symbols show the results of the numerical calculations (for numerical details see Sec. V) and it can be seen that they perfectly follow the predicted critical lines.

Figure 5(a) shows the two-dimensional system. For all curves with $p^{\text {(site) }}<1$, we have $3 \Theta s>1, N_{\text {crit }}^{\text {(site) }}>N_{\text {crit }}^{\text {(bond) }}$ and the corresponding critical lines are therefore identical with the appropriate dashed lines. For the case $p^{(\text {site })}=1$, we have $3 \Theta s<1$ and therefore the solid and the dashed lines cross at $D_{\text {crit }}$ to form a combined critical line. Figure 5(b) shows systems with $N=5$ that possess much smaller values of $s$, due to their higher number of neighbors. Again, we have $3 \Theta s<1$ and the critical line is composed of the full red line and the appropriate dashed line. Whenever the red line determines the phase transition, the dependence between $N_{\text {crit }}$ and $\langle D\rangle$ becomes linear, due to the pure linear relation (7). We

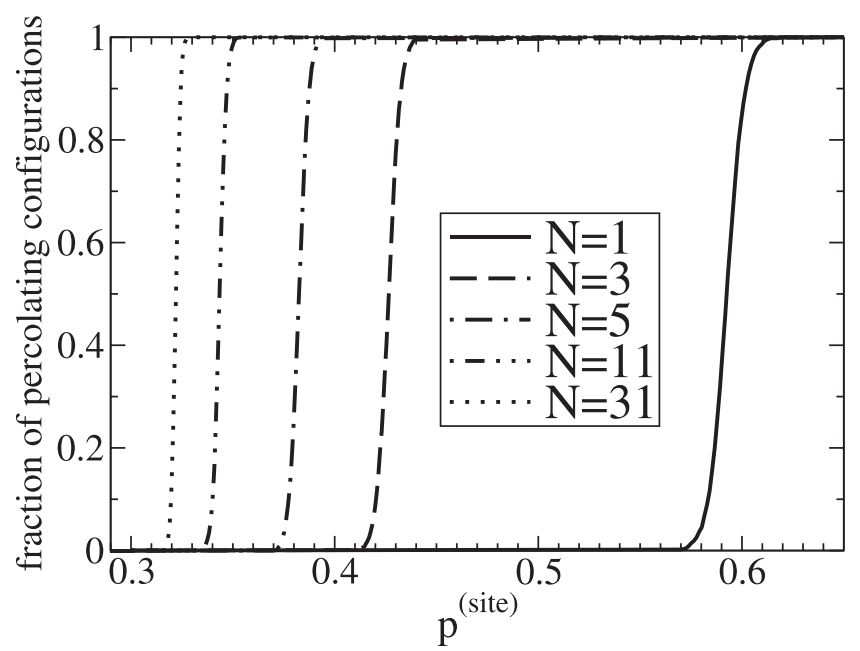

FIG. 4. Numerical simulations for the site percolation threshold $p_{c}^{(\text {site })}(N)$ : fraction of percolating systems versus occupation probability. The straight line represents the site percolation threshold for an ideal $2 \mathrm{~d}$ film and the interrupted lines approximate $3 \mathrm{~d}$ films of $N=3,5,11$, and 31 monolayers.

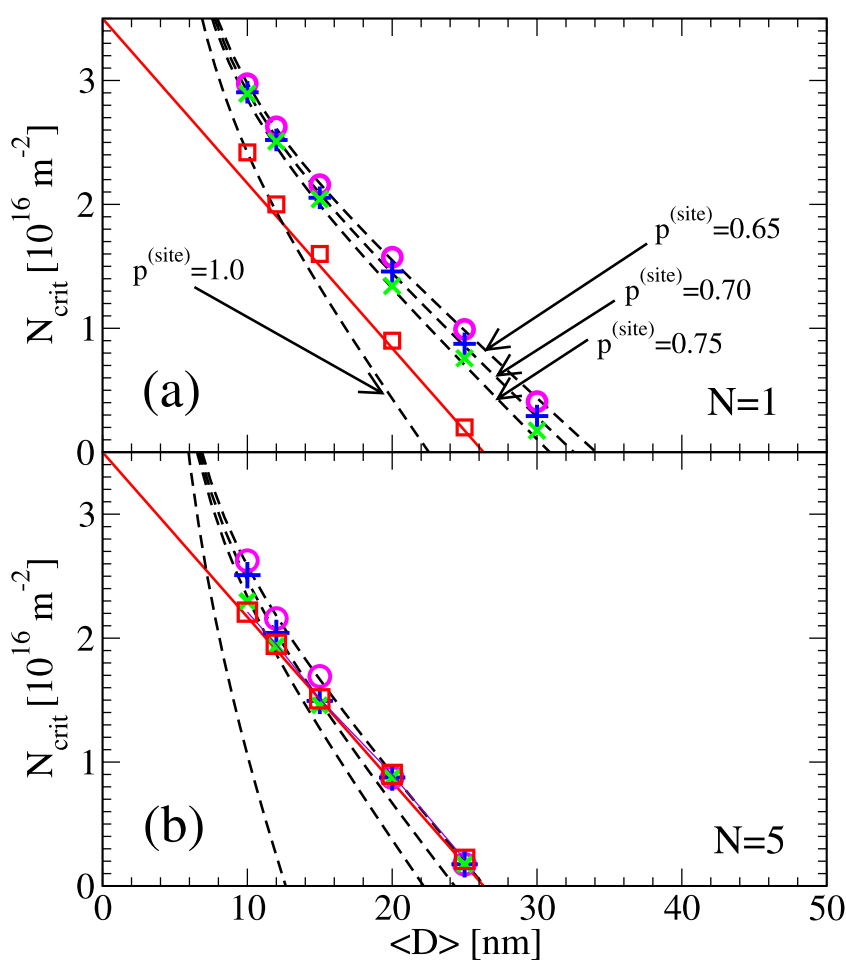

FIG. 5. Critical surface gas density $N_{\text {crit }}$ versus the mean grain diameter $\langle D\rangle$ for systems with (a) $N=1$ and (b) $N=5$ layers. In each figure, the full (red) line represents Eq. (7) with slope $\Theta N_{D} / 2$, independent of $p^{\text {(site) }}$. The dashed lines show the theoretical predictions of Eq. (9) that depend on $p^{\text {(site) }}$ while the symbols show the corresponding numerical simulations. The values for $\langle k\rangle$ (which here depend on $N$ and $\left.p^{\text {(site) }}\right)$ are: (pink) circles $\left(p^{(\text {site })}=0.65\right)$ : $\langle k\rangle \simeq 2.67$ for $N=1$ and $\langle k\rangle \simeq 3.65$ for $N=5$, (blue) pluses $\left(p^{\text {(site) }}=\right.$ $0.70):\langle k\rangle \simeq 2.84$ for $N=1$ and $\langle k\rangle \simeq 3.93$ for $N=5$, (green) crosses $\left(p^{(\text {site })}=0.75\right):\langle k\rangle \simeq 3.02$ for $N=1$ and $\langle k\rangle \simeq 4.20$ for $N=5$ and (red) squares $\left(p^{(\text {site })}=1.0\right):\langle k\rangle \simeq 4$ for $N=1$ and $\langle k\rangle \simeq 6$ for $N=5$.

assume that this is the underlying reason for the linear dependence of $N_{\text {crit }}$ on $\langle D\rangle$ found in former works on polydisperse systems ${ }^{14}$ (except for very small $\langle D\rangle$ ) which had not been fully understood before.

\section{MONTE-CARLO SIMULATIONS}

We performed numerical simulations for both the monodisperse and the polydisperse systems. The diameters of the grains are taken from a truncated Gaussian distribution

$$
P(D)= \begin{cases}C \exp \left[-\frac{1}{2}\left(\frac{D-\langle D\rangle}{\delta D}\right)^{2}\right], & |D-\langle D\rangle|<\gamma\langle D\rangle \\ 0, & |D-\langle D\rangle|>\gamma\langle D\rangle\end{cases}
$$

with the width $\delta D$ and the corresponding normalization constant $C$. The truncation is introduced in order to exclude certain unrealistic grain contacts (the center of a grain should not lie inside another grain). With $\gamma \approx 0.66$ and $\delta D=\langle D\rangle / 4$ which we used in our simulations, the truncated tails refer to $18 \%$ of the total Gaussian integral. In addition to the variation of grain sizes in polydisperse systems, the positions of the grain centers are randomly shifted away from the lattice points and the individual exposed surfaces $S_{\exp }^{(i)}$ are explicitly taken into account.

Equations (7)-(9) describe the monodisperse systems, but both equations are quite universal and contain all 
relevant information that can be gained from our model (as already explained above). We therefore expect the specific shape of the characteristic curves to be maintained to a large extent, even in the case of disorder. In the following sections, we want to see in which way the characteristic curves $\mu\left(N_{r}\right)$ are affected by the disorder and will therefore compare the monodisperse to the polydisperse systems. Furthermore, we want to explore in which way useful sensor systems have to be designed for various purposes, with high or low sensitivity, with smooth characteristic curves or threshold behavior. Many applications require a continuous reading without any threshold value. However, in other cases mentioned in the introduction, a predetermined threshold is desirable that can be tuned by a proper choice of $\Theta$ or the system parameters that determine $D_{\text {crit }}$ and $s$. Therefore, we now put our focus on the dependence of the sensor properties to the different microscopical parameters that can be designed on purpose: grain size, number of layers, fragility of the network (percolative structure), and polydispersity. To illustrate the behavior of the various sensors in a most systematic way, we show in the following sections the characteristic curves by always varying one system-parameter and keeping the others fixed.

\section{A. Influence of the mean grain size $\langle D\rangle$}

In Fig. 6, we show the characteristic curves for different mean grain-sizes $\langle D\rangle$ by keeping the number $N$ of layers and the occupation probability $p^{\text {(site) }}$ and thus the coordination number $\langle k\rangle$ fixed $\left(N=11\right.$ and $\left.p^{(\text {site })}=0.43\right)$. The different symbols (and colors) in the figure refer to different values of $\langle D\rangle$, while open and filled symbols indicate the monodisperse and the polydisperse systems, respectively. For the largest grain size, no gas-induced conductance switching is present ( $\mu$ is always large) but for all other grain sizes the conductance switching is clearly visible. As in Fig. $3, N_{\text {crit }}^{(\text {site }}$ is indicated by the arrows, while $N_{\text {crit }}^{(\text {bond })}$ roughly coincides with the beginning of the appropriate dashed curves (precisely for $\mu \rightarrow 0$ ). In accordance with Eqs. (7) and (9), the

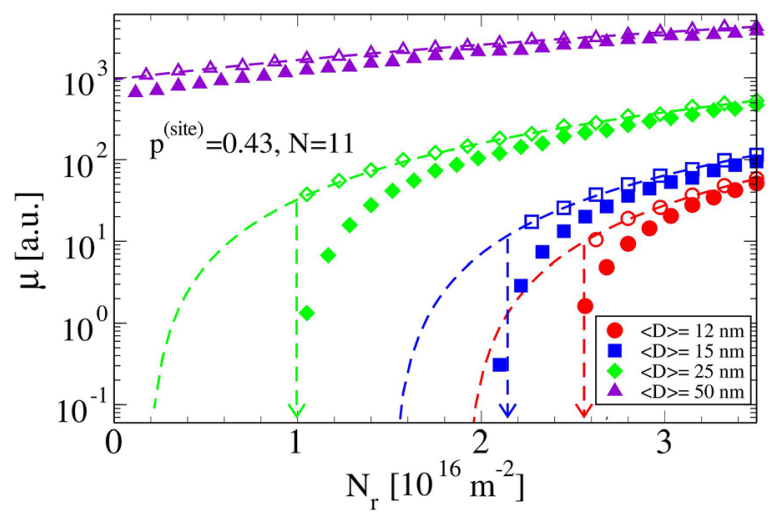

FIG. 6. Mobility $\mu$ (that corresponds to the diffusion constant $D_{s}$ ) versus surface density $N_{r}$ for various mean grain-sizes $\langle D\rangle$ for fixed number of layers $N=11$ and occupation probability $p^{(\text {site })}=0.43$ leading to the coordination number $\langle k\rangle \simeq 2.69$. Open and filled symbols represent the numerical simulations of the monodisperse and the polydisperse systems, respectively. The dashed lines represent the theoretical prediction of Eq. (8). As in Fig. 3(a), $N_{\text {crit }}^{(\text {site }}>N_{\text {crit }}^{(\text {bond })}$ where $N_{\text {crit }}^{\text {(site) }}$ is indicated by the arrows and $N_{\text {crit }}^{(\text {bond })}$ by the beginnings of the dashed curves. critical gas density $N_{\text {crit }}$ increases with decreasing grain-size $\langle D\rangle$ and $N_{\text {crit }}^{(\text {site })}>N_{\text {crit }}^{(\text {bond })}$ for all monodisperse systems, i.e., $N_{\text {crit }}=N_{\text {crit }}^{(\text {site })}$. As a result, the characteristics of the monodisperse system (open symbols) which lie perfectly on the theoretical curve, as proposed by Eq. (8) (dashed lines), do not follow this line till the bottom, but show a sudden jump at $N_{r}=N_{\text {crit }}^{(\text {site })}$.

The polydisperse systems (filled symbols) show a very similar behavior. Most remarkably, the conductance switching takes place close to the value of $N_{\text {crit }}=N_{\text {crit }}^{\text {(site) }}$ of the monodisperse systems. For large values of $N_{r}$ far above $N_{\text {crit }}$, the characteristics of both systems approach each other towards a common asymptotic value $\mu\left(N_{\mathrm{ox}}\right)$ (on the logarithmic scale) that scales with $\langle D\rangle^{3}$. Only for small values of $N_{r}$, the filled symbols do not follow the dashed lines, but clearly lie below. We will show later that site percolation effects due to variable grain sizes that are absent in monodisperse systems are responsible for this behavior. This leads to a much steeper increase of $\mu$ for the polydisperse systems close to the detection limit, namely to a much higher sensitivity. Hence, in the case $N_{\text {crit }}^{\text {(site) }}>N_{\text {crit }}^{\text {(bond) }}$, the broad distribution of the grain sizes is the important parameter to achieve a large sensitivity. We will see in the following figures that the differences in the sensitivity between mono- and polydisperse systems vanish in the case of $N_{\text {crit }}^{\text {(site) }}<N_{\text {crit }}^{\text {(bond) }}$.

\section{B. Influence of the coordination number $\langle\boldsymbol{k}\rangle$}

In Fig. 7, we have varied the occupation probability $p^{(\text {site })}$ and thus the coordination number $\langle k\rangle$ by keeping all other parameters fixed $(\langle D\rangle=15 \mathrm{~nm}, N=11)$. Again, $N_{\text {crit }}^{(\text {site }}$ is indicated by the arrows, while $N_{\text {crit }}^{(\text {bond) }}$ can roughly be recognized by the beginning of the dashed curves. While $N_{\text {crit }}^{\text {(bond }}$ does not depend on $p^{\text {(site) }}$ (see Eq. (7)), $N_{\text {crit }}^{\text {(site) }}$ decreases with increasing $p^{\text {(site) }}$ (see Eq. (9)) so that both cases, $N_{\text {crit }}=N_{\text {crit }}^{(\text {site }}$ and $N_{\text {crit }}=N_{\text {crit }}^{\text {(bond) }}$ are now possible. Fig. 7 shows that for systems where $p^{(\text {site) }}$ is small (i.e., close to $p_{c}^{\text {(site) }}(N)$ ), $N_{\text {crit }}=N_{\text {crit }}^{(\text {site })}$ and therefore a sudden jump of the conductance

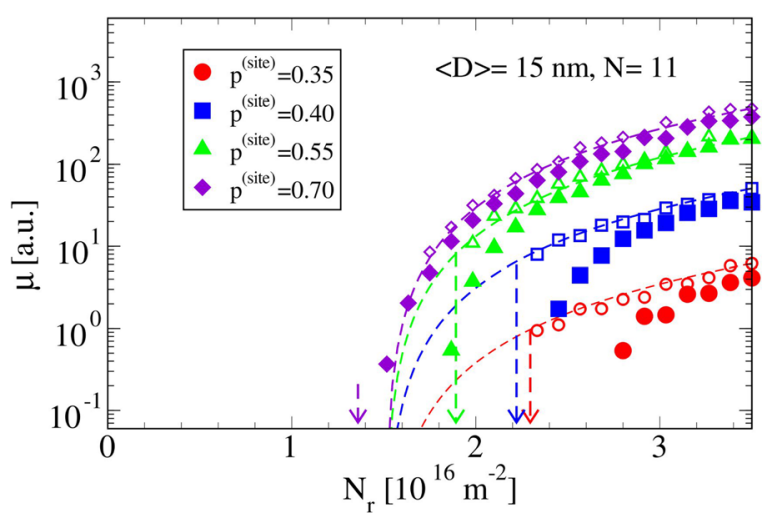

FIG. 7. Mobility $\mu$ versus surface density $N_{r}$ for fixed mean grain size $\langle D\rangle=15 \mathrm{~nm}$ and number of layers $N=11$ and varying occupation probability $p^{\text {(site) }}$. The meanings of the open (filled) symbols, dashed lines, and the arrows are the same as in Fig. 6. Note that both cases $N_{\text {crit }}^{\text {(site) }}>N_{\text {crit }}^{\text {(bond) }}$ and $N_{\text {crit }}^{(\text {site }}<N_{\text {crit }}^{(\text {bond })}$ occur in this figure. 
(start of the symbols) at $N_{r}=N_{\text {crit }}$ occurs. Again, the open symbols of the monodisperse systems follow the dashed lines (theoretical calculations of Sec. IV), while the filled symbols of the polydisperse systems lie below indicating site percolation effects. For increasing values of $p^{(\text {site })}$, on the other hand, $N_{\text {crit }}=N_{\text {crit }}^{\text {(bond) }}\left(\right.$ which can be seen for $p^{(\text {site })}=0.7$ ) and all symbols of the mono- and polydisperse systems follow the dashed line from the beginning. This indicates that even the polydisperse system is fully described by the theory for the simplified systems where no percolation effects have been taken into account. As we will explain at the discussion of Fig. 9, the reason of this counterintuitive behavior is that close to $N_{\text {crit }}$ the strengthening of the bonds superimposes all percolation effects.

Fig. 7 also shows that with increasing $p^{(\text {site })}$ the characteristic curves gradually approach each other towards one common mastercurve. This behavior is in accordance with Eq. (8), since $\mu\left(N_{\mathrm{ox}}\right)$ converges towards the conductance of a homogenous system for $p^{(\text {site })} \rightarrow 1$.

\section{Influence of the number of layers $\mathbf{N}$}

In Fig. 8, we vary the number $N$ of layers, while $p^{(\text {site })}=0.65$ and $\langle D\rangle=15 \mathrm{~nm}$ are kept fixed. Again, $N_{\text {crit }}$ is determined by the competition between $N_{\text {crit }}^{(\text {(site) }}$ and $N_{\text {crit }}^{(\text {bond }}$ where the latter is kept fixed so that, as in the foregoing subsection, the ratio $N_{\text {crit }}^{\text {(site) }} / N_{\text {crit }}^{\text {(bond) }}$ differs for each curve. For (very) small $N$-values, $N_{\text {crit }}=N_{\text {crit }}^{\text {(site) }}>N_{\text {crit }}^{\text {(bond) }}$, while for larger $N$-values $N_{\text {crit }}^{(\text {site }}$ approaches $N_{\text {crit }}^{\text {(bond) }}$ for the chosen parameters. While in Fig. $7, N_{\text {crit }}^{\text {(site) }}$ has been varied by $p^{(\text {site })}$, it is now modified by $N$ via $p_{c}^{(\text {site) }}(N)$ (see Eq. (9)).

With increasing $N$, the characteristic curves again collapse onto one mastercurve reflecting the rapid convergence of $p_{c}^{(\text {site) }}(N)$ towards the $3 \mathrm{~d}$ percolation threshold (see Fig. 4). At the same time, also the characteristic curves for the mono- and polydisperse systems approach each other which means that again the increase (strengthening) of the existing bonds (present in both systems) obviously superimposes the bond-percolation effect of the

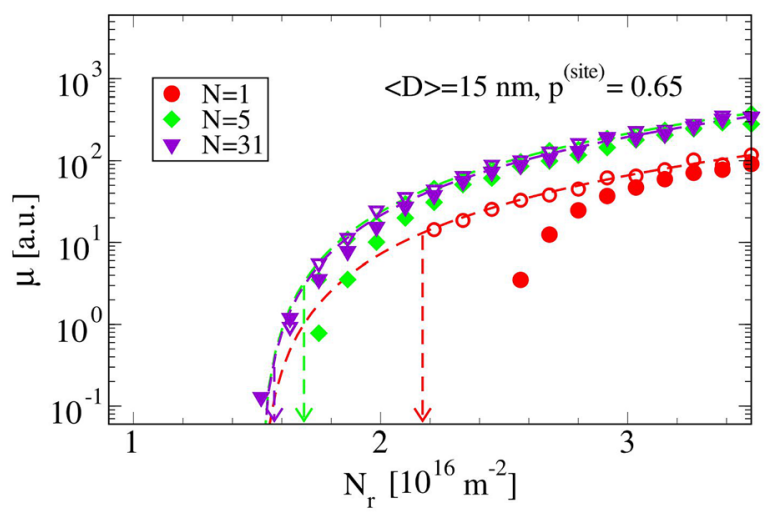

FIG. 8. Mobility $\mu$ versus surface density $N_{r}$ for fixed mean grain size $\langle D\rangle=$ $15 \mathrm{~nm}$ and occupation probability $p^{(\text {site })}=0.65$ and varying number of layers $N$. The meanings of the open (filled) symbols, dashed lines, and the arrows are the same as in Fig. 6.

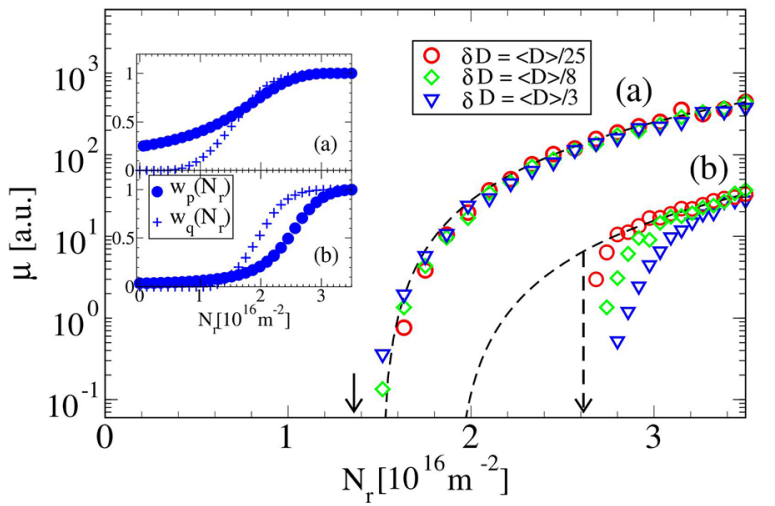

FIG. 9. Characteristics for two different systems with the parameters $\langle D\rangle=15 \mathrm{~nm}, N=11$, and $p^{(\text {site })}=0.7$ (a) and $\langle D\rangle=12 \mathrm{~nm}, N=3$, and $p^{\text {(site) }}=0.48$ (b). The grain sizes are taken from Eq. (12) with $\delta D=\langle D\rangle / 25$ (red circles), $\delta D \simeq\langle D\rangle / 8$ (green diamonds), and $\delta D \simeq\langle D\rangle / 4$ (blue triangles). While for system (a) the occupation probability $p^{\text {(site) }}$ is much larger than the critical density $p_{c}(N=11) \simeq 0.3221$, for system (b) $p^{(\text {site })}$ is very close to $p_{c}(N=3) \simeq 0.4266$ (see Fig. 4). The dashed line shows the theoretical prediction for monodisperse systems (see Eq. (6)), the arrows mark $N_{\text {crit }}^{\text {(site) }}$ according to Eq. (9). Inset: Fraction $w_{p}\left(N_{r}\right)$ of conducting grains (full circles) and fraction $w_{q}\left(N_{r}\right)$ of conducting bonds (crosses) of the total number of grains and bonds of the whole structure for $\delta D \simeq\langle D\rangle / 3$ and for both systems (a) and (b).

polydisperse system. A noticeable deviation from the mastercurve only occurs for the parameter range of $p^{(\text {site })} / p_{c}^{(\text {site })}(N) \simeq 1$. For the systems of fixed values of $p^{(\text {site })}$ shown here, this corresponds to very small $N$-values, most pronounced for $N=1$, i.e., for 2 d-systems (circles). For $N=1$, the shapes of the characteristic curves of the monoand polydisperse systems differ strongly. In particular, the polydisperse system shows the steeper increase and thus a higher sensitivity. We will see in Subsection VD that again, this is due to percolation effects.

\section{Influence of the polydispersity}

In Fig. 9, we explore the influence of the width $\delta D$ of the distribution of grain-sizes (Eq. (12)) on the characteristic curves of the polydisperse systems. For small values of $\delta D$, the systems are expected to approach the monodisperse systems, while percolation effects could play a role at larger values of $\delta D$. For a systematic viewpoint, we choose two typical sets of system-parameters which, for the corresponding monodisperse system (that still serves as a guideline) lead to the two cases (a) $N_{\text {crit }}^{(\text {bond) }}>N_{\text {crit }}^{\text {(site) }}$ and (b) $N_{\text {crit }}^{\text {(bond) }}<N_{\text {crit }}^{(\text {(site) }}$. In order to analyze the role of possible percolation effects in more detail, we also estimate the percentage of conducting grains $w_{p}\left(N_{r}\right)$ and of conducting bonds $w_{q}\left(N_{r}\right)$ as a function of $N_{r}$ for the broadest value of $\delta D=\langle D\rangle / 3$ for both cases (a) and (b) (see insets of Fig. 9). For the chosen parameter sets, see caption of Fig. 9.

For case (a), where $N_{\text {crit }}^{(\text {site })}<N_{\text {crit }}^{\text {(bond) }}$ holds, we can see in the corresponding inset (a) that close to $N_{\text {crit }}$ for the polydisperse systems the values of $w_{q}\left(N_{r}\right)$ (crosses) lie (slightly) below those of $w_{p}\left(N_{r}\right)$ (circles) which shows that the bonds become conducting at a higher values of $N_{r}$ than the grains. This means that also for the polydisperse systems the bonds 
play the crucial role. Figure 9 shows that in this case, the characteristic curves for different $\delta D$ do not show noticeable changes with increasing $\delta D$ but collapse onto a single mastercurve. Most interestingly, the symbols of the mono- and polydisperse systems follow the dashed line over the whole range of $N_{r}$ leading to a continuous increase of $\mu$ over several orders of magnitude. This data-collapse coincides with the prediction of Eq. (8) which has been derived for the monodisperse system and only takes the strengthening of the grain contacts into account. This means that the pronounced increase of $\mu$ stems from the strengthening of the grain contacts that superimpose possible percolation effects which therefore do not influence the shape of the characteristic curve, but only form an inherent feature.

In order to detect percolation effects, we have to focus on case (b) where for the corresponding monodisperse system $N_{\text {crit }}^{\text {(bond }}<N_{\text {crit }}^{\text {(site) }}$ applies and the critical gas density therefore is determined by $N_{\text {crit }}^{\text {(site) }}$, i.e., by the conductance switching of the grains. The corresponding inset (b) shows that now, for the polydisperse systems, the values of $w_{p}\left(N_{r}\right)$ lie well below those of $w_{q}\left(N_{r}\right)$, which shows that the bonds become conducting at a lower value of $N_{r}$ than the grains with the result that site percolation effects should now be dominant. Several differences to system (a) can be seen in the figure: First, the symbols start at much higher values of $N_{r}=N_{\text {crit }}^{\text {(site) }}$, in full agreement with all results of the foregoing subsections, where $N_{\text {crit }}^{\text {(bond }}<N_{\text {crit }}^{\text {(site) }}$ applied. Second, with increasing $\delta D$ large deviations from Eq. (8) (dashed line) occur, which means that the occurring site percolation effects, in contrast to the bond-percolation effects in system (a), clearly affect the shape of the characteristic curves (see also Fig. 6). As a consequence, close to $N_{\text {crit }}$ the conductance values drop rapidly with increasing $\delta D$, leading to an increase of the measurable $\mu\left(N_{r}\right)$-range and to a better resolution at quite large values of $N_{r}$ that is not achieved by the curves of case (a).

In summary, our findings suggest that for the polydisperse systems with small mean grain-sizes and high porosity, i.e., in the case $N_{\text {crit }}^{\text {(bond) }}<N_{\text {crit }}^{\text {(site) }}$, a site percolation effect takes place close to $N_{\text {crit }}$ which stems from the loss of grains due to the lack of charge carriers. For systems with larger grains and lower porosity where $N_{\text {crit }}^{\text {(site) }}<N_{\text {crit }}^{(\text {bond) }}$, the conductance switching originates from the influence of the bonds.

We can compare these findings to the 2d-simulations of Fig. 3(a) in Ref. 14, where the system parameters for lower $\langle k\rangle$-values lead to $N_{\text {crit }}^{(\text {site) }}>N_{\text {crit }}^{(\text {bond) }}$ (see Eqs. (7) and (9)). In this range, also our calculations suggest that site percolation effects are dominant. For the characteristic curve of Ref. 14 with the highest coordination number $\langle k\rangle=4$, on the other hand, $N_{\text {crit }}^{(\text {site })} \approx N_{\text {crit }}^{\text {(bond) }}$ holds. Accordingly, this system is probably already fully described by the variation of the bonds (see Eq. (8)) and the steep increase of the characteristic curves for $\langle k\rangle=4$ is not a site percolation effect, but reflects the strengthening of the grain contacts which is attended by an inherent bond-percolation effect. Also, the slopes in Fig. 3(b) of Ref. 14 can be well explained by the corresponding parameters $\Theta N_{D} / 2$ and $N_{D} /(6 \mathrm{~s})$ of Eqs. (7) and (9), respectively (see Ref. 15), explaining the linear dependence of $N_{\text {crit }}$ on $\langle D\rangle$, except for very small $\langle D\rangle$ (see also above).

\section{SUMMARY AND OUTLOOK}

We have modeled films of sintered metal oxide nanoparticles and have calculated the conductance (via the electron mobility) by random walk simulations taking into account the possibility that ultrasmall nanograins can be insulating and that bonds between conducting grains can be cleaved leading to site- and bond-percolation effects, respectively. Our numerical and analytical analysis reveals that the characteristic curves are determined by the interplay of two relevant thresholds for the density of reducing gas, $N_{\text {crit }}^{\text {(site) }}$ and $N_{\text {crit }}^{\text {(bond) }}$ that depend in a well-defined way on the system parameters. Additionally, the grain size distribution can have considerable influence on the shape of the characteristic curves.

So far, all currently available commercial surface sensitive gas sensors show characteristic curves that rise continuously with the target gas concentration. However, in many automatic applications, legal thresholds have to be obeyed to release an alarm or prealarm. Up to now, these systems consist of a sensor with a continuous characteristic curve, an amplifier, and an electronic comparator to cause an action. Such automatic systems would become more reliable and easier to produce and maintain if the sensor element itself had a switching characteristic at a preset concentration at the corresponding legal threshold value. Up to now, gas sensors with intrinsic switching behavior are not realized as products. However, switching due to the percolation effect is realized in a compound material, a polymer filled with small metal balls, forming a conductive path. At a predefined threshold current the thermal expansion of the polymer interrupts the current flow. Since many years these reversible fuses are produced in high numbers.

We believe that our findings help to understand the theoretical background needed to design high-performing gas sensors with intrinsic detection thresholds. For systems with $N_{\text {crit }}^{\text {(site) }}>N_{\text {crit }}^{\text {(bond) }}$ where the grains become insulating prior to the bonds, we show that for monodisperse systems a significant jump of the conductance from the basic level to the first signal value occurs at the threshold value $N_{\text {crit }}^{\text {(site) }}$. In polydisperse systems, namely with a broad distribution of grain sizes, the described conductance jump diminishes by the site-percolation effect. This means that the characteristic curve starts at smaller conductance values, but shows a steeper increase which corresponds to an enhanced sensitivity. The morphological conditions which lead to the condition $N_{\text {crit }}^{\text {(site) }}>N_{\text {crit }}^{\text {(bond) }}$ are met in highly porous systems consisting of small grains with strongly sintered bonds. In order to obtain a high surface to volume rate, it is advantageous to consider films of several monolayers $N$ due to the lower percolation threshold in these systems in comparison to $2 \mathrm{~d}$-systems.

The condition $N_{\text {crit }}^{\text {(site) }}<N_{\text {crit }}^{\text {(bond) }}$, on the other hand, leads to a continuous increase of the overall conductance which mainly reflects the increase of the bonds. This situation can be achieved by weakly connected grains, larger grains, and 
systems with large coordination number. In these systems, the exposed surface is hardly big enough to obtain insulated grains, even when covered with oxygen. Polydispersity in these systems leads to bond-percolation effects which, at least for the realistic sintering strength considered in this paper, have no detectable influence on the characteristic curves.

In summary, we have found that three competing mechanisms, namely, site percolation, bond percolation, and the increasing conductance values of existing bonds are needed to understand the conductance switching in semiconducting nanoparticle systems on exposure to a reducing gas. On the basis of these findings, we proposed a framework to design gas sensors with intrinsic detection thresholds and sketched system morphologies necessary to achieve these goals.

\section{ACKNOWLEDGMENTS}

We gratefully acknowledge financial support from the Deutsche Forschungsgemeinschaft and valuable discussions with Jörg Hennemann and Benjamin Becker.

${ }^{1}$ G. Heiland, Sens. Actuators 2, 343 (1982).

${ }^{2}$ D. Kohl, J. Phys. D 34, R125 (2001).

${ }^{3}$ T. Wagner, C.-D. Kohl, M. Fröba, and M. Tiemann, Sensors 6, 318 (2006).

${ }^{4}$ M. Tiemann, Chem.-Eur. J. 13, 8376 (2007).

${ }^{5}$ T. Waitz, B. Becker, T. Wagner, T. Sauerwald, C.-D. Kohl, and M. Tiemann, Sens. Actuators B 150, 788 (2010).

${ }^{6}$ M. K. Kennedy, F. E. Kruis, H. Fissan, B. R. Mehta, S. Stappert, and

G. Dumpich, J. Appl. Phys. 93, 551 (2003).

${ }^{7}$ D. Kohl, Sens. Actuators 18, 71 (1989).
${ }^{8}$ V. V. Sysoev, T. Schneider, J. Goschnick, I. Kiselev, W. Habicht, H. Hahn, E. Strelcov, and A. Kolmakov, Sens. Actuators B 139, 699 (2009).

${ }^{9}$ X. Wang, S. S. Yee, and W. P. Carey, Sens. Actuators B 24-25, 454 (1995).

${ }^{10}$ C. Xu, J. Tamaki, N. Miura, and N. Yamazoe, Sens. Actuators B 3, 147 (1991).

${ }^{11}$ Z. Jin, H.-J. Zhou, Z.-L. Jin, R. F. Savinell, and C.-C. Liu, Sens. Actuators B 52, 188 (1998).

${ }^{12}$ M. Bögner and T. Doll, in Advanced Gas Sensing, edited by T. Doll (Kluwer Academic Publishers, Dordrecht, 2003), Chap. 1, p. 2.

${ }^{13}$ M. Ulrich, C.-D. Kohl, and A. Bunde, Thin Solid Films 391, 299 (2001).

${ }^{14}$ M. Ulrich, A. Bunde, and C.-D. Kohl, Appl. Phys. Lett. 85, 242 (2004).

${ }^{15}$ In our simulations, we assumed for the surface density of initially adsorbed oxygen $N_{\text {ox }}$ and for the donor density $N_{r}$ the same values as used in Ref. 14: $N_{\text {ox }}=3.5 \times 10^{16} \mathrm{~m}^{-2}$ and $N_{D}=3.5 \times 10^{24} \mathrm{~m}^{-3}$.

${ }^{16}$ K. D. Benkstein, N. Kopidakis, J. van de Lagemaat, and A. J. Frank, J. Chem. Phys. 107, 7759 (2003).

${ }^{17}$ A. Ofir, S. Dor, L. Grinis, A. Zaban, T. Dittrich, and J. Bisquert, J. Chem. Phys. 128, 064703 (2008).

${ }^{18}$ A. Solbrand, H. Lindström, H. Rensmo, A. Hagfeldt, S.-E. Lindquist, and S. Södergren, J. Chem. Phys. B 101, 2514 (1997).

${ }^{19}$ G. Korotcenkov, Mater. Sci. Eng. R 61, 1 (2008).

${ }^{20}$ B. Sapoval, S. Russ, and J.-N. Chazalviel, J. Phys.: Condens. Matter 8, 6235 (1996).

${ }^{21}$ Fractals and Disordered Systems, 2nd ed., edited by A. Bunde and S. Havlin (Springer, Berlin, 1996); D. Stauffer and A. Aharony, Introduction to Percolation Theory, 2nd ed., (Taylor and Francis, London, 1994).

${ }^{22}$ S. Havlin and D. ben-Avraham, Adv. Phys. 51, 187 (2002); A. Bunde and J. Draeger, Phys. Rev. E 52, 53 (1995).

${ }^{23}$ In principle, a small transition regime follows the point $N_{r} \geq N_{\text {crit }}^{(\text {site })}$, where the fraction of conducting grains increases still further (from $p_{c}^{(\text {site) }}(N)$ to 1 ), according to Eq. (3). However, as the width of this $N_{r}$-regime is only of the order $0.5 \times 10^{15} \mathrm{~m}^{-2}$, i.e., very narrow as compared to the relevant range of $N_{r}$, we describe the characteristic curve by Eq. (8), i.e., by the change of the bonds.

${ }^{24}$ T. Sauerwald and S. Russ, "Percolation effects in metal oxide gas sensors and related systems," in Gas Sensing Fundamentals, edited by C.-D. Kohl and T. Wagner (Springer, Heidelberg, 2013). 